\title{
OS CAMINHOS DA FONOAUDIOLOGIA NO SISTEMA ÚNICO DE SAÚDE - SUS
}

\author{
The ways of the speech-language therapy \\ in the Unique System of Health - SUS
}

\author{
Mirna Dorneles Moreira ${ }^{(1)}$, Helena Bolli Mota ${ }^{(2)}$
}

\section{RESUMO}

Tema: os caminhos da Fonoaudiologia no Sistema Único da Saúde - SUS. Objetivo: realizar um breve relato da evolução das questões de saúde no Brasil e da evolução do serviço de Fonoaudiologia no Sistema Público além de realizar uma breve análise sobre a importância de pesquisas nesta área. Conclusão: o serviço de Fonoaudiologia vem apresentando avanços significativos no Sistema Único de Saúde. Desde sua inserção no SUS, entre as décadas de 70 e 80, muitos conceitos e práticas têm sido reavaliados. No entanto, é preciso que se tenha conhecimento desta caminhada para que seja possível fazê-la avançar cada vez mais. Acredita-se que este serviço merece importante atenção das ações de saúde pública e que são necessárias evidências científicas que comprovem a importância deste trabalho.

DESCRITORES: Patologia da Fala e Linguagem; Saúde Pública; Pesquisa nos Serviços de Saúde; Uso da Informação Científica na Tomada de Decisões em Saúde

\section{INTRODUÇÃO}

A Fonoaudiologia como ciência busca o bemestar do indivíduo e da sua comunidade. Para atingir-se esta meta é fundamental o conhecimento dos caminhos percorridos até o momento para que se possa entender o presente e planejar um futuro melhor.

O serviço de Fonoaudiologia no Sistema Único de Saúde vem crescendo com o passar dos anos. Muitos conceitos e práticas têm sido reavaliados com o intuito de se oferecer um serviço de qualidade e de acordo com os preceitos da saúde pública. No entanto, ainda há muito a melhorar. Neste artigo pretende-se mostrar os caminhos da Fonoaudiolo-

(1) Fonoaudióloga; Secretaria Municipal de Saúde de Rosário do Sul, Rosário do Sul, RS; Mestre em Distúrbios da Comunicação Humana pela Universidade Federal de Santa Maria.

(2) Fonoaudióloga; Professora Adjunta do Curso de Fonoaudiologia e do Curso de Mestrado em Distúrbios da Comunicação Humana da Universidade Federal de Santa Maria, UFSM, Santa Maria, RS; Doutora em Linguística Aplicada pela Pontifícia Universidade Católica do Rio Grande do Sul.

Conflito de interesses: inexistente gia no Sistema Único de Saúde para que, através desta compreensão, seja possível avançar nesta caminhada.

As questões de saúde no Brasil datam do final do século $X I X$, início do século $X X{ }^{1}$. Surgem como questão social durante a economia cafeeira. Em 1892, Emílio Ribas inicia o combate à febre amarela em São Paulo, seguido por Osvaldo Cruz, a partir de 1903. Ambos motivados pela organização assistencial e pela exclusão dos pobres à assistência médica, que ocorria na época. Em 1923 é criado o Departamento Nacional de Saúde Pública, quando deveria ocorrer a centralização e transformação das questões de saúde em problema nacional. No entanto, prosseguem as soluções de caráter local. No início da década de 30 inicia-se a Medicina Previdenciária, a partir da criação de Institutos de Aposentadorias e Pensões, e o Estado passa a responder as questões sociais de forma mais abrangente. Como a Medicina Previdenciária centrava suas ações em hospitais, sendo estas as principais ações de prestações de serviços de saúde, os problemas de saúde da população de baixa renda, crescem de forma significativa. Para tentar solucionar estes problemas, é criado em 1953, o Ministé- 
rio da Saúde, responsável, em todo o país, pelos serviços de tuberculose, malária, lepra, câncer, entre outros, e educação sanitária, fiscalização da medicina e bioestatística. Em 1967 é criado o Instituto Nacional de Previdência Social (INPS), onde a assistência médica torna-se obrigatória a todos os trabalhadores atuantes e seus dependentes ${ }^{2}$.

Em 1977 é criado o Instituto Nacional de Assistência Médica da Previdência Social (INAMPS), que tinha por objetivo a assistência médica aos trabaIhadores urbanos, aos servidores civis da União e suas autarquias e do Distrito Federal. Nessa época, a assistência médica às classes mais abastadas era prestada pela iniciativa privada, aos pobres, pela igreja e outras entidades filantrópicas e à classe trabalhadora oficial, pelo INAMPS. Os Municípios atendiam as urgências e as campanhas e os programas de erradicação e/ou controle de determinados agravos ficavam a cargo, principalmente, dos órgãos governamentais federais e estaduais ${ }^{3}$.

Enquanto esses acontecimentos ocorriam no Brasil, a Organização Mundial da Saúde (OMS) realizava em 1978, em Alma-Ata (antiga URSS), a Conferência Internacional sobre Cuidados Primários de Saúde, com a participação de inúmeros países, inclusive o Brasil. Nesta Conferência surge a definição de saúde como "um estado de completo bem-estar, de equilíbrio biopsicossocial e não somente a ausência de doenças ou enfermidades" e várias propostas são sugeridas com o intuito de se alcançar Saúde para Todos no Ano $2000{ }^{1}$.

No final da década de 70 , início de 80 , o setor saúde passa por uma grave crise no Brasil. Cria-se, então, o Programa Nacional de Serviços Básicos de Saúde (PREV-SAÚDE), que tinha como proposta a reorganização e ampliação dos Serviços de Saúde, incluindo saneamento e habitação, regionalização, hierarquização, participação da comunidade, entre outros, considerando que o primeiro atendimento deveria ser a porta de entrada do Sistema de Saúde ${ }^{2,3}$.

Convênios começam a serem assinados, em 1983, com as Secretarias de Saúde, estaduais e municipais, e em 1984 são implantadas as Ações Integradas de Saúde (AIS). Com base nas propostas da AIS e nas discussões realizadas durante a VIII Conferência Nacional de Saúde - 1986, em 1987 é assinado o Sistema Único e Descentralizado de Saúde (SUDS), e com a publicação da Constituição de 1988 defini-se o Sistema Único de Saúde (SUS). Em 1992, a IX Conferência Nacional de Saúde exige o cumprimento da Lei do SUS ${ }^{2,3}$.

Deste modo, a Constituição de 1988 surge como um marco no campo da saúde pública ${ }^{4}$. De acordo com esta Constituição o Sistema Único de Saúde (SUS) foi definido como uma rede regionalizada de ações e serviços que visam "o acesso universal e igualitário da população para a promoção, proteção e recuperação de sua saúde", tendo como princípios fundamentais: a equidade, a universalidade e a integralidade. A equidade caracteriza-se por oferecer oportunidades iguais, em termos de tratamento, para necessidades iguais. A universalidade seria a garantia de atendimento a todo e qualquer cidadão. A integralidade seria uma atenção integral à saúde (prevenção primária, secundária e terciária) ${ }^{3}$.

Prevenção não se limita à diminuição da ocorrência de doenças. Dela devem fazer parte conteúdos que visem promover, proteger, diagnosticar, tratar e reabilitar a saúde individual e coletiva ${ }^{5}$.

A prevenção pode ser dividida em três fases: prevenção primária: prevenir a ocorrência de doenças ou disabilidades antes que elas aconteçam; prevenção secundária: reverter um quadro em andamento, alternando ou retardando sua evolução, e prevenção terciária: atividades de vigilância e manutenção do potencial subjacente depois do episódio patológico, para minimizar complicações e disabilidades ${ }^{6}$. Transferindo esses níveis para o contexto da Fonoaudiologia pode-se citar como uma medida de prevenção primária a promoção e orientação do aleitamento materno, como uma intervenção em nível secundário o diagnóstico e tratamento dos desvios fonológicos e como uma medida em nível terciário a reabilitação de um sujeito afásico ${ }^{7}$.

A década de 90 trouxe inúmeros benefícios para o setor da saúde no Brasil, uma vez que em setembro de 1990 foi assinada a lei 8080, conhecida como "Lei Orgânica da Saúde", que definiu o SUS Sistema Único de Saúde. Em dezembro do mesmo ano foi assinada a lei 8142, que dispõe sobre a participação da comunidade na gestão do SUS, sobre as transferências intergovernamentais de recursos financeiros e estabelece as Conferências de Saúde e o Conselho de Saúde como instâncias colegiadas do SUS. O SUS tem por objetivos: identificar e divulgar os fatores condicionantes e determinantes da saúde, formular políticas de saúde destinadas à redução de riscos de doenças e de outros agravos e ao acesso igualitário e universal às ações e aos serviços de saúde, dar assistência às pessoas por intermédio de ações de promoção, proteção e recuperação da saúde ${ }^{8}$.

É necessário que o fonoaudiólogo assim como os demais funcionários do Sistema Único de Saúde - SUS - tenham conhecimento de seus preceitos, características administrativas e sua repercussão em um contexto mais amplo. O fonoaudiólogo precisa se inteirar dos assuntos pertinentes ao SUS a fim de poder organizar seu trabalho e direcionar ações, que surtam efeito na instituição pública e na comunidade ${ }^{7}$. Deve se manter informado sobre as 
propostas das políticas públicas do Ministério da Saúde conforme as variadas faixas etárias, saúde infantil, saúde escolar e do adolescente, saúde mental, saúde de idoso, etc.

Deste modo, o objetivo desta pesquisa é realizar um breve relato da evolução das questões de saúde no Brasil e da evolução do serviço de Fonoaudiologia no Sistema Público, além de realizar uma breve análise sobre a importância de pesquisas nesta área.

\section{MÉTODOS}

As ferramentas de busca para esta pesquisa de revisão de literatura foram o Medline e o Lilacs por intermédio da pesquisa avançada simultânea de base de dados da biblioteca virtual em saúde - BVS da Bireme, além de livros com conteúdos históricos, monografias e dissertações. Procurou-se resgatar um pouco da história da Fonoaudiologia no SUS e dar ênfase à importância da fundamentação científica relacionada ao serviço de Fonoaudiologia no SUS. Foram utilizados os seguintes descritores: patologia da fala e da linguagem, fonoaudiologia e serviço público, evolução da fonoaudiologia no Sistema Único de Saúde, evolução das questões de saúde no Brasil, Sistema Único de Saúde, pesquisa nos serviços de saúde. A revisão bibliográfica do tipo histórica foi realizada entre os meses de março de 2006 até julho de 2008 e é um recorte de uma dissertação de mestrado. Utilizou-se como critério de inclusão para a seleção das referências a adequação ao tema pesquisado, excluindo-se aquelas que não se encaixavam com exatidão ao tema em questão.

\section{REVISÃO DA LITERATURA}

\section{A evolução do serviço de Fonoaudiologia no Sistema Público}

Foi entre a década de 70 e 80 que os fonoaudiólogos iniciaram suas atividades no sistema público, alguns via secretarias de educação, outros pelas secretarias de saúde. Nesse período, nem todas as pessoas tinham acesso à saúde. Somente as classes mais abastadas. Porém, aos poucos a classe trabalhadora foi conquistando este direito que se concretizou com a criação do INAMPS em $1977^{3}$. Nessa época o número de profissionais era pequeno e os trabalhos isolados, sem nenhum tipo de integração e sem propostas abrangentes. Este fato comprometeu a efetividade do trabalho e não surtiu efeito na comunidade em geral e muito menos aos olhos dos órgãos competentes. Os procedimentos eram voltados para estrutura de consultório, devido ao fato da formação reabilitadora que o profissional de Fonoaudiologia recebia. Era difícil propor e organizar serviços voltados para grandes populações. O serviço, então, se concentrava em ambulatórios de saúde mental e hospitais ${ }^{1}$, mantendo a proposta reabilitdora.

$\mathrm{Na}$ segunda metade da década de 80 , com a promulgação da Constituição de 88 , define-se 0 Sistema Único de Saúde e a população brasileira passa a ter acesso universal e igualitário para a promoção, recuperação e proteção da sua saúde ${ }^{3}$. Como fruto desta conquista surgem, principalmente em São Paulo, os concursos públicos para as Secretarias de Saúde para a contratação de fonoaudiólogos. Desta forma, muitos destes profissionais foram lotados nos centros de saúde - porta de entrada do sistema - iniciando neste momento a inserção da Fonoaudiologia na atenção primária à saúde. Porém, manteve-se a estrutura de consultório, gerando insatisfação tanto do fonoaudiólogo quanto da instituição pública. A partir daí, o fonoaudiólogo começou a organizar seus ambulatórios, levando em consideração a pressão da demanda reprimida e seus conhecimentos anteriores, atendendo às diversas patologias da comunicação, dando ênfase especial ao fato de que casos mais significativos requerem atendimentos especiais $e$ na maioria das vezes, multidisciplinaridade. Deste modo, precisou estudar e começar a trabalhar com alguns conceitos de epidemiologia, objetivando o levantamento das patologias de maior ocorrência na população, assim como suas características e possibilidades de organização ambulatorial ${ }^{1}$.

Surgem então, no início dos anos 80 , as primeiras pesquisas mostrando a ocorrência de patologias da comunicação na população brasileira. Mais tarde, pesquisas mostram que do ponto de vista fonoaudiológico, em geral, a população que apresenta maior demanda de manifestação primária é a infantil ${ }^{9}$, concordando com estatísticas americanas que mostram que, segundo o Committee on Language, Speech and Hearing Problems, as patologias da comunicação constituem a primeira causa de disabilidades infantis ${ }^{10}$. Segundo a "American Speech, Language and Hearing Association" (ASHA), são consideradas como desordens da comunicação as alterações de fala (articulação, voz e fluência), da linguagem (forma, conteúdo e função comunicativa) e da audição (sensibildade, função, processamento e fisiologia) ${ }^{10-12}$. Porém, os dados de morbidade fonoaudiológica ainda são muito precários.

A partir deste momento os fonoaudiólogos sentem o desejo e a necessidade de formalizar sua inserção nos demais serviços da instituição e estes tentam organizar propostas de ações a serem executadas junto aos programas de pediatria e pueri- 
cultura, saúde do adolescente, saúde da mulher, do trabalhador e do idoso, além da sua inserção em creches e escolas da área de abrangência das unidades, mostrando que a Fonoaudiologia na saúde pública também evoluiu, assim como as questões de saúde no Brasil.

Outro aspecto muito importante a ser considerado é que as ações desenvolvidas em uma unidade de porta de entrada do sistema não são individuais, e sim coletivas. Por isto deve-se ter muito cuidado, porque as coletividades não são iguais, o que é adequado para uma comunidade nem sempre o é para outra. Daí a necessidade de conhecimento da área de abrangência de trabalho para a realização de ações pertinentes às necessidades do local.

Paralelamente a este processo, as Universidades vão adequando suas grades curriculares, visando preparar melhor o fonoaudiólogo para esta nova área de atuação.

O fonoaudiólogo que atua no SUS deve ser um generalista, capaz de identificar as questões fonoaudiológicas de maior relevância na sua comunidade de abrangência, capaz de elaborar e efetivar ações que visem uma solução, adotando medidas preventivas sempre que possível. Também deve ser capaz de organizar um ambulatório de atendimento que se identifique com a sua unidade de saúde, visando sempre a qualidade no atendimento à população ${ }^{1}$. Além disso, para que o fonoaudiólogo consiga desenvolver um trabalho de acordo com os preceitos da saúde pública é fundamental que ele tenha conhecimento dos assuntos pertinentes ao SUS?.

Atualmente acredita-se que o papel do fonoaudiólogo tornou-se mais amplo, sendo possível projetar ações e prevenções cabíveis a um determinado grupo, desde que este seja bem conhecido ${ }^{13}$.

Defende-se que os aspectos de fala, da linguagem e da audição devem ser considerados atributos da saúde uma vez que suas manifestações patológicas abalam a competência e o desempenho comunicativo verbal e não verbal, intra e interpessoal. Eles geram sofrimento, mesmo não causando dor física, não apresentando sinais e sintomas expressos laboratorialmente, não sendo levados à cura por meio de ingestão de drogas nem levando à morte. Porém, essas manifestações patológicas limitam a capacidade do ser humano criar e transformar o mundo, através do poder da palavra, gerando um enorme impacto na experiência pessoal e comprometendo a qualidade de vida. É necessária uma intervenção voltada para impedir e/ou para romper o processo da doença, sendo este o primeiro passo para produzir saúde, global e fonoaudiológica ${ }^{6}$. Como a habilidade comunicativa é um dos maiores contribuintes para a qualidade de vida, toda a ação preventiva nesta área irá favorecer este processo ${ }^{14}$.

Deste modo, as atividades de saúde pública, sejam elas de promoção, proteção ou recuperação, devem estar voltadas à problematização das questões do cotidiano ${ }^{15}$. Algumas propostas baseadas em promoção da saúde têm sido apresentadas a fim de se (re)pensar e (re)direcionar as ações públicas ${ }^{16,17}$.

Acredita-se que a descentralização do sistema de saúde possibilita um planejamento mais específico e de acordo com as necessidades da população de determinada região. Isto, em tese, ajudaria a melhorar a qualidade dos serviços de saúde prestados à população e atenderia de forma mais eficaz suas necessidades ${ }^{18}$.

Torna-se importante reconhecer as características de demanda específicas em cada comunidade e sua área de abrangência. Após este reconhecimento, se estabelece um corpus de ações e programas com caráter preventivo, quanto ao uso de medidas que evitem ou minimizem os distúrbios de comunicação pertinentes a cada realidade social. Essas ações e serviços necessitam de constantes avaliações a fim de garantir a prevenção e tratamento das problemáticas da comunicação ${ }^{8}$.

A comunicação, sendo objeto de estudo da Fonoaudiologia, merece importante atenção das ações de saúde pública, uma vez que possibilita ao indivíduo se colocar como agente transformador da sociedade e da sua realidade. No entanto, é preciso apresentar evidências científicas sobre a importância deste trabalho e das transformações decorrentes destas intervenções ${ }^{19}$. Ou seja, é necessário fortalecer a Fonoaudiologia baseada em evidências, comprovando a importância das nossas ações através de publicações científicas.

Reforçando esta ideia, autores salientam que a Fonoaudiologia necessita investir em conhecimento científico para fundamentar o crescimento da atividade profissional e que a atenção dispensada à Fonoaudiologia voltada para uma visão preventiva e coletiva ainda é deficiente ${ }^{20}$.

Dados confirmam que as pesquisas desenvolvidas no campo da saúde pública ainda são escassas, principalmente quando comparadas com a produção por áreas específicas da Fonoaudiologia ${ }^{21}$.

Quanto às políticas públicas, há referências que o SUS surgiu como uma alternativa para a universalização do atendimento à população, tendo como prioridade a prevenção, porém ainda necessita de muitos investimentos nesta área. O SUS pretendia oferecer um atendimento eficiente e comprometido com a qualidade de vida da população, porém as dificuldades para se atingir este fim são inúmeras. 
No entanto, com o enfrentamento das dificuldades e com a aplicação de políticas públicas bem definidas em todas as esferas do governo voltadas aos interesses da sociedade é possível melhorar o desenvolvimento e o fortalecimento no atendimento à saúde ${ }^{22}$.

Existe a necessidade da inserção de políticas públicas na Fonoaudiologia, além de que a maior parte dos fonoaudiólogos ainda não tem formação e treinamento para efetivar um trabalho preventivo. Com o intuito de suprir esta dificuldade, os novos currículos já estão em fase de adaptação e as expectativas futuras para a prevenção em Fonoaudiologia são cada vez melhores ${ }^{23}$.

Os avanços alcançados pelo movimento da reforma sanitária brasileira são evidentes, do mesmo modo que são relevantes os percalços e as dificuldades na luta por melhores condições de saúde e de trabalho em saúde em nosso país. No decorrer das últimas quatro décadas, muitas pessoas têm trabalhado para fazer valer o direito à saúde assim como para desenvolver estratégias técnicas e políticas capazes de tornar tal direito efetivo ${ }^{24}$. Atualmente encontram-se fonoaudiólogos inseridos em alguns programas do Ministério da Saúde, como PSF - Programa Saúde da Família, saúde infantil, saúde mental, saúde escolar e do adolescente, etc ${ }^{25}$. O Ministério da Saúde também tem investido em políticas públicas abrangendo a saúde auditiva, a saúde da pessoa idosa, os núcleos de apoio à saúde da família - NASF, etc ${ }^{26}$.

Como a demanda no setor de Fonoaudiologia é muito grande e na maioria das vezes, é maior que a disponibilidade de recursos humanos, tecnológicos ou financeiros, entre outros, torna-se imprescindível priorizar necessidades ${ }^{27}$.

\section{CONCLUSÃO}

A Fonoaudiologia na Saúde Pública tem apresentado crescimento e vários estudos já demonstraram a elevada ocorrência de distúrbios da comunicação em pessoas que recorrem aos serviços públicos. Acredita-se que chegou o momento de se fazer um levantamento do que está sendo feito em termos de prevenção e tratamento dos distúrbios da comunicação. É preciso, pois, tomar providências para suprir a alta demanda e adequar os serviços à realidade atual. Isto requer maior número de fonoaudiólogos atuando na Saúde Pública, profissionais preparados para lidar com Saúde Pública e mais atenção do Governo ao que se refere às políticas públicas em Fonoaudiologia.

Atualmente, a atenção dispensada à Fonoaudiologia voltada para uma visão preventiva e coletiva ainda é deficiente. Precisa-se investir em pesquisas nessa área a fim de fundamentar a importância destas ações.

\begin{abstract}
Background: the ways of the speech-language therapy in the Unique System of Health - SUS. Purpose: to accomplish a brief report about the health questions evolution in Brazil and speechlanguage therapy service evolution in the Public System, in addition to carry through a quick analysis on the concern of research in this area. Conclusion: speech-language therapy service has been presenting significant advances in the Unique System of Health. Since its insertion in SUS, between the decades of 70 and 80 , many conceptions and practices have been reevaluated. However, it is necessary to have acquaintance on this step so that we can make it to advance each time more. We believe that this service deserves important attention from the actions of public health and that scientific evidences are necessary in order to prove the significance of this work.
\end{abstract}

KEYWORDS: Speech-Language Pathology; Public Health; Research in Health Services; Use of Scientific Information for Health Decision Making 


\section{REFERÊNCIAS}

1. Befi D. A inserção da fonoaudiologia na atenção primária à saúde. In: Befi $\mathrm{D}$, organizador. Fonoaudiologia na atenção primária à saúde. São Paulo: Lovise; 1997. p. 15-36.

2. Puccini RF. Saúde pública: histórico e conceitos básicos. In: Vieira RM, organizador. Fonoaudiologia e saúde pública. Carapicuíba: Pró-Fono; 1995. p.3-22.

3. Garbin W. O sistema de saúde no Brasil. In: Vieira RM, organizador. Fonoaudiologia e saúde pública. Carapicuíba: Pró-Fono; 1995.p.24-34.

4. Gomes EMGP, Remencius NR. Fonoaudiologia na Unidade Básica de Saúde. In: Largota MGM, César CP. A fonoaudiologia nas instituições. São Paulo: Lovise; 1997. p. 183-86.

5. Andrade CRF. Fonoaudiologia preventiva: teoria e vocabulário técnico científico. São Paulo: Lovise; 1996.

6. Andrade CRF. Fases e níveis de prevenção em fonoaudiologia: ações coletivas e individuais. In: Vieira RM, organizador. Fonoaudiologia e saúde coletiva. 2. ed. Carapicuíba: Pró-Fono; 2000. p. 81-104.

7. Goulart BNG. A fonoaudiologia e suas inserções no sistema único de saúde: análise prospectiva. Rev Fonoaudiol Bras. 2003; 2(4):29-34.

8. Beltrame IL. Fonoaudiologia e saúde pública. In: Oliveira ST, organizador. Fonoaudiologia hospitalar. São Paulo: Lovise; 2003. p.18-27.

9. Andrade CRF, Lopes DMB, Wertzner HF. Uma reflexão sobre a fonoaudiologia preventiva. Ciênc Cultur. 1991; 43(7):152-3.

10. Committee on Language, Speech and Hearing Problems. Prevention: a challenge for the profession. ASHA (EUA). 1984; 26(8):35-7.

11. Committee for Children and Youths with Communication Disorders. Inclusive practices. Am Speeh-Lang Hear Assoc. 1996; 35:44.

12. Committee on Language, Speech and Hearing Association. Comunicative disorders and variations. ASHA. 1982; 24(11):9-12.

RECEBIDO EM: 01/09/2007

ACEITO EM: 24/06/2008

Endereço para correspondência:

Mirna Dorneles Moreira

Rua Barão do Cerro Largo, 1486

Rosário do Sul - RS

CEP: 97590-000

E-mail: mirna@ rosulonline.com.br
13. Guedes ZCF. A atuação do fonoaudiólogo e seu papel na escola. In: Befi D, organizador. Fonoaudiologia na atenção primária à saúde. São Paulo: Lovise; 1997. p.119-38.

14. McGinnis JM. Prevention. ASHA (EUA). 1984; 26(6):22-4.

15. Lopes SMB. Cultura, linguagem em fonoaudiologia: uma escuta do discurso familiar no contexto da Saúde Pública. [dissertação]. São Paulo (SP): Universidade de São Paulo; 2001.

16. Czeresnia D. The concept of health and the difference between prevention and promotion. Cad Saúde Públ. 1999; 15(4):701-9.

17. Eakin J, Mclean H. A critical perspective on research and knowledge development in health promotion. Canad J Public Health. 1992; 83(Supl1):72-6.

18. Goulart BNG. As idiossincrasias do sistema único de saúde. [monografia]. Porto Alegre (RS): Universidade Federal do Rio Grande do Sul; 2001. 19. Souza RPF, Cunha DA, Silva HJ. Fonoaudiologia: a inserção da área de linguagem no Sistema Único de Saúde (SUS). Rev CEFAC. 2005; 7(4):426-32.

20. Costa AG, Cavalheiro LG. Queixas fonoaudiológicas em comunidades da cidade de Salvador, Bahia. In: XIV Congresso Brasileiro de Fonoaudiologia, 4 a 7 de outubro de 2006. Rev Soc Bras Fonoaudiol - Suplemento Especial. Salvador, BA, 2006. [CD-ROM].

21. Perdigão JCA, Lemos SMA. Produção científica em Saúde Publica: análise retrospectiva dos anais dos Congressos Brasileiros de Fonoaudiologia. In: XIV Congresso Brasileiro de Fonoaudiologia, 4 a 7 de outubro de 2006. Rev Soc Bras Fonoaudiol - Suplemento Especial. Salvador, BA, 2006. [CD-ROM].

22. Fortes AMC. Fonoaudiologia: uma abordagem pública. [monografia] Santa Maria (RS): Universidade Federal de Santa Maria; 2006.

23. Danieli LC. Fonoaudiologia Preventiva. [monografia] Santa Maria (RS): Universidade Federal de Santa Maria; 2006.

24. Mendes VLF. Uma clínica no coletivo: experimentações no Programa de Saúde da Família. São Paulo: Hucitec; 2007. p. 25.

25. Brasil. Programas de Saúde do Ministério da Saúde. Disponível em: URL: http://www.misau.gov. $\mathrm{mz} / \mathrm{pt} /$ programas-de-saude. Acesso em 02 jul 2008. 26. Brasil. Ministério da Saúde. Portaria № 389 de 3 de março de 2008. Disponível em: URL: http:// dtr2001.saude.gov.br/sas/PORTARIAS/Port2008/ GM/GM-389.htm. Acessado em 02 julho 2008.

27. Goulart BNG, Chiari BM. Construção e aplicação de indicadores de saúde na perspectiva fonoaudiológica: contribuições para reflexão. Rev Soc Bras Fonoaudiol. 2006; 11(3):194-204. 